\title{
Polysaccharide-Supported Planar Bilayer Lipid Model Membranes ${ }^{\dagger}$
}

\author{
Tobias Baumgart and Andreas Offenhäusser* \\ Max-Planck Institute for Polymer Research, Ackermannweg 10, D-55128 Mainz, Germany
}

Received J une 28, 2002. In Final Form: September 19, 2002

\begin{abstract}
Bilayer lipid membranes were deposited onto two different thin water swellable polymer cushions predominantly by Langmuir-Blodgett trough methods. Membranes consisting of zwitterionic lipids supported by agarose films were shown to be unstable, as observed by fluorescence mi croscopy, reflection interference contrast mi croscopy, and theimpossi bi lity of bilayer spreading (Rädler ,J .; Strey, H.; Sackmann, E. Langmuir 1995, 11, 4539-4548) on the agarose surface. Chitosan, formerly observed to permanently coat liposome surfaces of zwitterionic lipid membranes (Henriksen, I.; Smistad, G.; Karlsen, J . Int. J . Pharm. 1994, 101, 227-236), was used to prepare thin films by spin-coating and was found to sufficiently adsorb and thereby stabilize planar bilayer lipid membranes. Bilayer spreading on a chitosan surface was observed, indicating the self-healing ability of chitosan-supported lipid membranes.
\end{abstract}

\section{Introduction}

Polymer interlayers havea great potential for effectively decoupling lipid membranes and the peripheral parts of integral membrane proteins from supports such as glass, gold, indium-tin oxide (ITO), or silicon devices. ${ }^{3-5}$ Therefore, numer ous efforts have been madeto prepare suitable polymer cushions, physisorbed or chemically coupled to an underlying support and physisorbed or chemically coupled to the lipid membrane on top.

Lipid membranes that areeither completely or partially chemically coupled to the polymer cushion are regarded to beadvantageous to membranestability. H owever, they increase the synthetic expense and considerably reduce membranefluidity. A highly fluid membraneenvironment is generally assumed to be favorable, if not essential, for the activity of membrane proteins. Therefore, in the present work, membranes were prepared which were purely physisorbed to the polymer cushion.

Ringsdorf's lab was probably the first to work on physisorbed polymer-supported lipid membranes on a sol id substrate. ${ }^{6-9}$ In theseworks, polyethyleneimine(PEI) was used as an interlayer between a solid support and an amphiphile membrane (see also refs 10 and 11). Ad-

* Corresponding author. Mailing address: Institute for Thin Films \& I nterfaces (ISG2), Forschungszentrum J ülich, D-52425 J ülich, Germany. Tel: +49-2461-61-2330. Fax: +49-2461-61-2333. E-mail: a.offenhaeusser@fz-juelich.de.

† Part of the Langmuir special issue entitled The Biomolecular Interface

(1) Rädler, J .; Strey, H.; Sackmann, E. Langmuir 1995, 11, 4539548.

(2) Henriksen, I.; Smistad, G.; Karlsen, J . Int. J . Pharm. 1994, 101, 227-236.

(3) Sackmann, E. Science 1996, 271, 43-48.

(4) Sackmann, E.; Tanaka, M. Tibtech 2000, 18, 58-64.

(5) Shen, W. W.; Boxer, S. G.; Knoll, W.; Frank, C. W. Biomacromolecules 2000, 2 (1), 70-79.

(6) Chi, L. F.;J ohnston, R. R.; Ringsdorf, H. Langmuir 1992, 8, 13601365.

(7) Chi, L. F .; ohnston, R. R.; Ringsdorf, H.; Kimizuka, N.; Kunitake,

T. Thin Solid Films 1992, 210, 111-113.

(8) Chi, L. F.; Anders, M.; Fuchs, H.; J ohnston, R. R.; Ringsdorf, H. Science 1993, 259, 213-216.

(9) Chi, L. F.; Fuchs, H.; J ohnston, R. R.; Ringsdorf, H. Thin Solid Films 1994, 242, 151-156.

(10) Wong, J. Y.; Majewski, J .; Seitz, M.; Park, C. K.; I sraelachvili, J. N.; Smith, G. S. Biophys. J . 1999, 77, 1445-1457. ditionally, the polysaccharides dextran, cellul ose $\mathrm{e}^{12-17}$ and polyacrylamide ${ }^{18,19}$ were applied to prepare polymersupported mono- and bilayer lipid membranes.

All of these systems share the fact that it is difficult or impossible to control or alter the membrane/substrate spacing by varying thethickness of theinterlayer polymer cushion. The control of the membrane/substrate spacing is of importance for surface sensitive techniques, like methods using evanescent fields. Additionally, the electrical analysis of single membrane channels requires a large ionic reservoir (i.e., a polymer cushion of sufficient thickness). ${ }^{20}$ The use of polymers, which aresoluble under particular conditions and otherwiseforma water-insoluble gel, is a way to avoid this problem. Both of the deposition methods, dip-coating and spin-coating, offer an extremely wide range of polymer thicknesses quite readily. Additionally, this approach is simpleand time-saving, which is favorable for extensive physicochemical investigations of polymer-cushioned lipid membranes. Agarose and chitosan are polysaccharides that can be dissolved under special conditions and are insol uble under the conditions used for membrane preparation and analysis.

Probably due to the simple fabrication of the polymer cushion, agar ose has been used extensively for supporting and thereby stabilizing black lipid membranes (BLMs), ${ }^{21-23}$

(11) Wong, J . Y.; Park, C. K.; Seitz, M.; I sraelachvili, J . Biophys. J 1999, 77, 1458-1468.

(12) Elender, G.; Kühner, M.; Sackmann, E. Biosens. Bioelectron. 1996, 11, 565-577.

(13) Raedler, J .; Sackmann, E. Curr. Opin. Solid State Mater. Sci. 1997, 2, 330-336.

(14) Györvary, E.; Wetzer, B.; Sleytr, U. B.; Sinner, A.; Offenhäusser, A.; Knoll, W. Langmuir 1999, 15, 1337-1347.

(15) Nissen, J .; Gritsch, S.; Wiegand, G.; Rädler, J . O. Eur. Biophys. I. B 1999, 10, 335-344.

(16) Hillebrandt, H.; Wiegand, G.; Tanaka, M.; Sackmann, E. Langmuir 1999, 15, 8451-8459.

(17) Wiegand, G.; J aworek, T.; Wegner, G.; Sackmann, E. Langmuir 1997, 13, 3563-3569.

(18) Arya, A.; Krull, U.J .; Thompson, M.; Wong, H. E. Anal. Chim. Acta 1985, 173, 331-336.

(19) Kühner, M.; Rampe, R.; Sackmann, E. Biophys. J . 1994, 67, 217-226.

(20) Costello, R. F.; Peterson, I. R.; Heptinstall, J .; Byrne, N. G.; Miller, L. S. Adv. Mater. Opt. Electron. 1998, 8, 47-52.

(21) Mueller, P.: Rudin, D. O.; Tien, H. T.; Wescott, W. C. Nature 1962, 194, 979

(22) Howard, R. E.; Burton, R. M. J . Am. Oil Chem. Soc. 1968, 45, $202-229$ 
which are obtained by the thinning of a lipid solution painted across a small aperture. BLM-like membranes were formed either on top of an agarose cushion exposed to electrolyte $e^{24-29}$ or sandwiched between two agarose sheets. 20,30,31 Additionally, monolayer lipid membranes weretransferred toagar osefilms by means of LangmuirBlodgett transfer ${ }^{32}$ Interestingly, agarose-supported BLM like membranes (i.e., li pid membranes coupl ed to a solventenriched lipid reservoir formed in an aperture) possess el ectrical properties which are comparable to thosefound for free-standing BLMs. ${ }^{24,25,27,28,33}$ It was shown ${ }^{29}$ that agarose-cushioned lipid bilayers allow the analysis of single ion channels, incorporated into the membrane. Other polymer-supported bilayer systems, however, showed el ectrical properties markedly different ${ }^{34-37}$ from those of free-standing BLMs, which are regarded to represent an electrically "perfect" membrane. In the present work, we evaluated whether it is possible to prepare bilayer membranes on agarose cushions by Langmuir-Blodgett trough methods.

As an alternativetoagarose, chitosan films wereapplied as membrane cushions. To the best of the authors' knowledge, this report is the first to describe the use of chitosan as a polymer cushion to support planar bilayer lipid membranes. However, chitosan has been utilized to coat liposomes with a monomolecular layer (so-called "chitosomes"), leading to an enhanced liposome stability. $2,38-40$ We demonstrate that chitosan polymer cushions, which are as easy to prepare as agarose films, have major advantages over agarosefor obtaining homogeneous and stable bilayer lipid membranes by LangmuirBlodgett trough methods and by bilayer spreading from a lipid reservoir.

\section{Methods and Materials}

FRAP/Fluorescence Microscopy. F or determining lateral self-diffusion coefficients, the method of fluorescence recovery

(23) Goldup, A.; Ohki, S.; Danielli, J . F. Recent Prog. Surf. Sci. 1970 3, 193-256.

(24) Hongyo, K.; J oseph, J .; Huber, R. J .; J anata, J . Langmuir 1987, 3, 827-830.

(25) Uto, M.; Araki, M.; Taniguchi, T.; Hoshi, S.; I noue, S. Anal. Sci. 1994, 10, 943-946.

(26) Hianik, T.; Dlugopolsky, J .; Gyeppessova, M.; Sivak, B.; Tien, H. T.; Ottova-Leitmannova, A. Bioel ectrochem. Bi oenerg. 1996, 39, 299302.

(27) Lu, X.; Ottova, A. L.; Tien, H. T. Bioel ectrochem. Bioenerg. 1996 39, 285-289.

(28) N ovotny, I.; Rhacek, V.; Tvarozek, V.; Nikolelis, D. P.; Andreou, V. G.; Siontorou, C. G.; Ziegler, W. Mater. Sci. Eng., C 1997, 5, 55-58. (29) Ide, T.; Yanagida, T. Biochem. Biophys. Res. Commun. 1999 265, 595-599.

(30) Costello, R. F.; Peterson, I. R.; Heptinstall, J .; Walton, D. J . Biosens. Bioelectron. 1999, 14, 265-271.

(31) Costello, R. F.; Evans, S. W.; Evans, S. D.; Peterson, I. R.; Heptinstall, J . Enzyme Microb. Technol. 2000, 26, 301-303.

(32) Dietrich, C.; Tampe, R. Biochim. Biophys. Acta 1995, 1238, 183191.

(33) Ziegler, W.; Gaburjakova, J .; Gaburjakova, M.; Sivak, B.; Rehacek, V.; Tvarozek, V.; Hianik, T. Colloids Surf., A 1998, 140, 357367.

(34) Lindholm-Sethson, B. Langmuir 1996, 12, 3305-3314.

(35) Sinner, A. Ph.D. Thesis, Technische Universität München, München, Germany, 1999.

(36) Cassier, T.; Sinner, A.; Offenhäusser, A.; Möhwald, H. Colloids Surf., B 1999, 15, 215-225.

(37) J enkins, A. T. A.; Hu, J .; Wang, Y. Z.; Schiller, S.; Foerch, R.; Knoll, W. Langmuir 2000, 16, 6381-6384.

(38) Henriksen, I.; Green, K. L.; Smart, J . D.; Smistad, G.; Karlsen,

J. Int. J. Pharm. 1996, 145, 231-240.

(39) Takeuchi, H.; Yamamoto, H.; Niwa, T.; Hino, T.; Kawashima, Y. Pharm. Res. 1996, 13, 896-901.

(40) Henriksen, I.; Vagen, S. R.; Sande, S. A.; Smistad, G.; Karlsen,

G. Int. J . Pharm. 1997, 146, 193-204. after photobleaching (FRAP) of membrane labels was used as described. ${ }^{14,41}$

The central part of the FRAP apparatus was an inverted microscope (IX-70, Olympus, Hamburg, Germany), equipped with a mercury burner (HBO 100, Olympus, 100 W) for wide-field illumination and an argon ion laser (Innova 90/4, Coherent, Dieburg, Germany), which was operated at a wavelength of 488 $\mathrm{nm}$ (at a power of $1.2 \mathrm{~W}$ ), for bleaching and fluorescence recovery measurements. Beam attenuation was performed by a combination of three pockels cells and four linear polarizers (Gsänger, Munich, Germany) in an alternating arrangement. Focus adjustment (and fluorescence microscopy) could be performed with the help of a light-enhancing camera (extended ISIS, Photonic Sciences, East Sussex, U.K.).

RICM. Reflection interference contrast microscopy (RICM) was performed as described in ref 42 . The central part of the setup was an inverted microscope (IX 70, Olympus; the same microscope allowed for fluorescence microscopy and F RAP with the same sample). A high-pressure mercury burner served as the light source. A band-pass filter ( $546 \mathrm{~nm}$, Zeiss, Göttingen, Germany) allowed for the selection of the intense line at 546.1 $\mathrm{nm}$ of the mercury lamp. A modified DIC cube (U-MDIC, Olympus) was equipped with two rotatable linear polarizers, needed for stray light suppression by means of the Antiflex technique. The microscope objective was a Zeiss Neofluar 63/ 1.25 (Zeiss, Göttingen, Germany). RICM images were recorded by means of a digital CCD camera (C 4742-95-12-NR, $1280 \times$ 1024 pixels; Hamamatsu, Germany) which was controlled by the software HiPic 32 (Hamamatsu, Germany).

Giant liposomes for interference microscopy were prepared from a lipid stock solution in chloroform, which was dried on a Teflon disk and desiccated under vacuum overnight. Subsequently, $100 \mathrm{mM}$ sucrose solution was added to the Teflon disk placed at the bottom of a glass beaker and left for at least $12 \mathrm{~h}$ at $40{ }^{\circ} \mathrm{C}$. During that time, the lipid swelled and formed closed vesicles. A few milliliters of this vesicledispersion wastransferred into a measurement chamber filled with $100 \mathrm{mM}$ inositol. The vesicles sedimented slowly to the bottom of the chamber due to the difference in density of sucroseinsidethe vesideand inositol outside. In the case of experiments with giant multilamellar vesicles (liposomes), the measurement chamber consisted of a Teflon frame $(10 \times 30 \times 40 \mathrm{~mm})$ into which a hole was drilled $(\varnothing 8 \mathrm{~mm})$. The Teflon frame was closed at the bottom by a glass coverslip.

Langmuir-Blodgett Trough Methods. Lipid bilayer membranes were obtained by two successive depositions of lipid monolayers on a Langmuir-Blodgett (LB) trough. Monolayers were obtained by spreading a lipid solution (chloroform, $1 \mathrm{mg} /$ $\mathrm{mL}$ ) on a Langmuir -Blodgett trough equipped with a mechanical dipper and Wilhel my balance (NI MA Technol ogy Ltd., Coventry, U.K.). Thesubphase consisted of ion-exchanged Milliporefiltered water (Millipore Milli-Q system, $R=18.2 \mathrm{M} \Omega \mathrm{cm}$ ). After the lipid solution was spread, the solvent was allowed to evaporate (for half an hour) and the film was compressed to the desired lateral pressure $\left(T=25^{\circ} \mathrm{C}\right)$. After compression, the film was equilibrated for another half an hour and finally deposited at a speed of $4 \mathrm{~mm} / \mathrm{min}$. The second monolayer was transferred by vertically pressing the substrate through the compressed monolayer at theair/water interface (Schäfer transfer). F or successful Schäfer transfers, the sample had to be dried thoroughly previously by means of a dry stream of $\mathrm{N}_{2}$, as otherwise the barrier position on the LB trough indicated an insufficient lipid transfer.

Lipids. The lipids DMPC (1,2-dimyristoyl-sn-glycero-3-phosphatidyl chol ine), egg-PC (a natural lipid mixture), and chol esterol (3 $\beta$-hydroxy-5-chol esten) wereobtained from Avanti Polar Lipids, Alabaster, $A L$, and used without purification. The following fluorescence probes were applied for membranelabeling: $\mathrm{N}$-(7nitrobenz-2-oxa-1,3-diazol-4-yl)-1,2-di hexadecanoyl-sn-glycero3-phosphoethanolamine, triethylammonium salt (NBD-PE), and 1, 1'-dioctadecyl-3,3,3',3'-tetramethylindocarbocyanine perchlorate (Dil), Molecular Probes, Leiden, The Netherlands. Fluo-

(41) Axelrod, D.; Koppel, D. E.; Schlessinger, J .; Elson, E.; Webb, W . W. Biophys. J . 1976, 16, 1055-1069.

(42) Rädler, J .; Sackmann, E. J . Phys. II France 1993, 3, 727-748. 
rescent lipids were added to thelipid solution at a concentration of $1 \mathrm{~mol} \%$ with respect to the host lipid. In all cases, fluorescent lipids were added to both bilayer leaflets.

Polymer Cushions. Chitosan and agarosewereobtained from Fluka/Sigma/Aldrich, Seelze, Germany, and used without purification. Chitosan was dissolved in a $1 \% \mathrm{v} / \mathrm{v}$ acetic acid solution $\left(99.8 \%\right.$, Riedel-de Haën, Seelze) ${ }^{43}$ at a concentration of $1 \% \mathrm{w} / \mathrm{w}$ by stirring overnight. Thesolutions werefiltered through syringe filters (Millex, Millipore Corp., Bedford, MA) with a pore size of $5 \mu \mathrm{m}$. Afterward, the solutions were centrifuged (Bi ofuge 22R, Heraeus, Germany) for $30 \mathrm{~min}$ at a speed of $11400 \mathrm{rpm}$. Thin chitosan films wereprepared by spin-coating thechitosan solution onto deaned, hydrophilic silicon wafers (Wacker Siltronic Corp. Stuttgart, Germany; thickness, $600 u \mathrm{~m} ;\langle 100\rangle$ orientation), which were cut into pieces of $25 \times 40 \mathrm{~mm}$. The wafers were thermally oxidized (IMM, Mainz; temperature, $\left.800{ }^{\circ} \mathrm{C}\right)$ to yield an oxide layer thickness of $160 \mathrm{~nm}$. In the case of RICM measurements, thepolymer layer was deposited ontotransparent glass substrates (Mettler Glas, Rettberg, Göttingen, Germany; length, $3.2 \mathrm{~cm}$; width, $2.6 \mathrm{~cm}$; thickness, $150-180 \mu \mathrm{m}$ ). Substrate cleaning was performed by sonication in a $2 \% \mathrm{v} / \mathrm{v} \mathrm{Hellmanex} \mathrm{solution} \mathrm{(Hellma,}$ Germany), followed by thoroughly rinsing the substrates in Millipore water. Spin-coating was typically performed at a spinning speed of $3000 \mathrm{rpm}$, which yielded film thicknesses around $140 \mathrm{~nm}$ in the dry state, as determined by ellipsometry To neutralize the films, the polymer-covered substrates were immersed for several hours in a borate buffer $(\mathrm{pH}=9.22$; M erck, Darmstadt, Germany) and afterward rinsed in Millipore water.

Agarosefilms were prepared by dipping dean substrates into a hot aqueous agarose solution of a concentration of $0.2 \% \mathrm{w} / \mathrm{w}$. Upon quickly withdrawing, a thin polymer film remained on the substrate surface and formed a thin gel film upon cooling. ${ }^{32}$

Vesicle F usion. Small unilamellar vesicles for vesiclefusion experiments were prepared by sonication. Lipid dissolved in chloroform was filled into test tubes and the solvent was evaporated while rotating the test tube so as to cover the inner walls by a thin lipid film. Subsequently, thelipid was thoroughly dried under vacuum overnight. Afterward, MilliQ water was added to the test tube and the lipid was allowed to rehydrate for at least $3 \mathrm{~h}$ at $40^{\circ} \mathrm{C}$. Sonication was performed by means of the tip sonicator Sonifier 250 (Branson, Danbury, CT), at a power of $30 \mathrm{~W}$ for $30 \mathrm{~min}$, using a water bath for cooling. The dear dispersion was centrifuged (L abofuge 200, Haraeus) at 5300 rpm for $15 \mathrm{~min}$, to remove sonicator debris. The vesicle dispersion was used directly after preparation. The dispersion was filled onto polymer-covered substrates and allowed to rest for several hours at a temperature of $30{ }^{\circ} \mathrm{C}$ (egg-PC) or $40{ }^{\circ} \mathrm{C}$ (DMPC). Afterward, the samples were washed by shaking them in pure MilliQ water.

\section{Results}

Bilayer lipid membranes were prepared on agaroseand on chitosan polymer films by sequentially depositing two lipid monolayers onto polymer-covered glass substrates. The first transfer was performed by Langmuir-Blodgett transfer and leads, in all cases, to homogeneous supported monolayer membranes that were stable over the time course of theexperiments (fluorescenceimages not shown).

The second monolayer was added by a Schäfer transfer.

Westudied theinfluence of themembranesupport type, the deposition temperature, and the membrane composition on membrane properties such as the following: homogeneity, stability, and self-healing (i.e., lateral bilayer membrane spreading), by fluorescence microscopy; the membrane/substrate interaction, by RICM ; and the possibility of vesicle fusion and the fluidity of the supported bilayer, by FRAP.

Fluorescence Microscopy. Agarose. Figure 1 shows that lipid membranes consisting of DMPC on agarose cushions were laterally structured, indicating a partial fragmentation of the deposited membrane. The bilayer

(43) Blair, H. S.; Huthrie, J .; Law, T.-K.; Turkington, P. J . Appl. Polym. Sci. 1987, 33, 641-656.

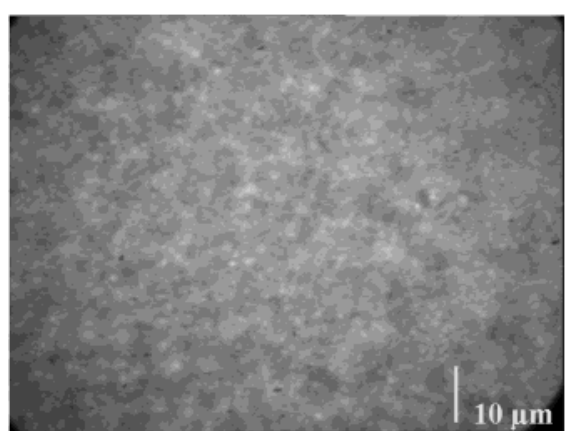

Figure 1. Fluorescence image of a DMPC bilayer membrane on an agarose cushion prepared by LB/LS transfer (lateral pressure, $35 \mathrm{mN} / \mathrm{m}$; deposition temperature, $25^{\circ} \mathrm{C}$ ), using fluorescence dye Dil, directly after transfer.

deposition at a transfer temperature of $15^{\circ} \mathrm{C}$ (below the main transition temperature of a DMPC bilayer, $\mathrm{T}_{\mathrm{t}}=23.8$ ${ }^{\circ} \mathrm{C}$ ) improved theinitial bilayer quality, but a temperature increase to $25^{\circ} \mathrm{C}$ again led to membrane fragmentation.

However, lipid bilayers of the same composition, deposited onto glass supports, al ways were found to show a perfectly homogeneous fluorescence at the resolution of the optical microscope and this state did not change with time (picture not shown).

One way to stabilize bilayer lipid membranes on solid substrates is the incorporation of cholesterol into the bilayer. The influence of cholesterol on the elastic properties of bilayers is well-known, ${ }^{44}$ and it has been found that homogeneous bilayers on planar dextran cushions could beobtained only if the membranecontained a considerable amount of cholesterol. ${ }^{19}$ This observation was explained ${ }^{45}$ by a reduction of undulations and/or by an interaction of the polar $\mathrm{OH}$-group of the cholesterol molecule with the underlying polysaccharide surface. Additionally, cholesterol compresses the membrane considerably, which enhances theel ectrical tightness of the bilayer. ${ }^{46} \mathrm{H}$ owever, in thecase of agarose-cushioned lipid bilayers, theaddition of chol esterol toDMPC membranes did not improvebilayer quality. I nstead, di rectly after deposition at a temperature of $15^{\circ} \mathrm{C}$, large vesicles were observed on the surface and in the surrounding medium beneath the supporting surface (Figure 2, left part). H owever, the comparison of Figure 2 with Figure 1 reveals that fluorescent (i.e., bilayer-covered) areas aremuch morehomogeneous when cholesterol is incorporated intothemembrane. Dewetting of the homogeneous bilayer parts could be observed within minutes after membrane preparation (Figure 2, right part).

Chitosan. Lipid bilayers supported by chitosan showed a much-improved initial bilayer quality directly after transfer (F igure3). A slight structuring was observed after storageat $35^{\circ} \mathrm{C}$ for several hours. Thelong-term stability of chitosan-cushioned membranes at increased temperatures could be increased even further by the addition of cholesterol. No decrease of membrane homogeneity was observed for DMPC membranes containing $30 \mathrm{~mol} \%$ of cholesterol during storage overnight at $35^{\circ} \mathrm{C}$. However, as in the case of agarose-cushioned membranes, a considerable influence of deposition temperature $\left(25^{\circ} \mathrm{C}\right.$ compared to $15^{\circ} \mathrm{C}$ ) on the initial bilayer quality was

(44) Sackmann, E. Bending Energy Concept of Vesicleand Cell Shapes and ShapeTransitions; Flyvbjerg, H., Ed.; Springer: Berlin, 1997; Vol. 480

(45) Kühner M. Ph.D. Thesis, Technische Universität München, München, Germany, 1996.

(46) Gritsch, S.; Nollert, P.; ähnig, F.; Sackmann, E. Langmuir 1998, 14, 3118-3125. 

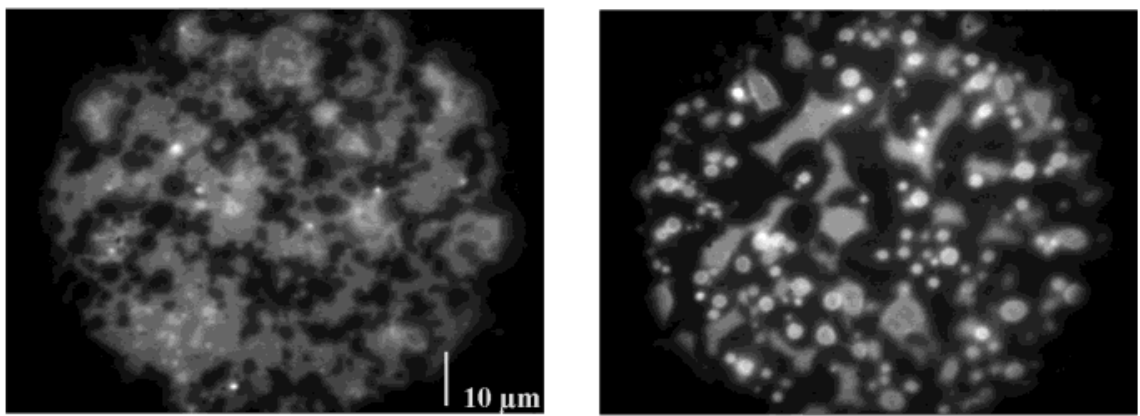

Figure 2. Fluorescence images of DMPC ( $30 \mathrm{~mol} \%$ cholesterol) bilayer preparations on agarose cushions. Deposition temperature and observation temperature in both pictures: $15^{\circ} \mathrm{C}$. Left picture, directly after transfer; right picture, 10 min after transfer (different location on the same sample as compared to the left picture).

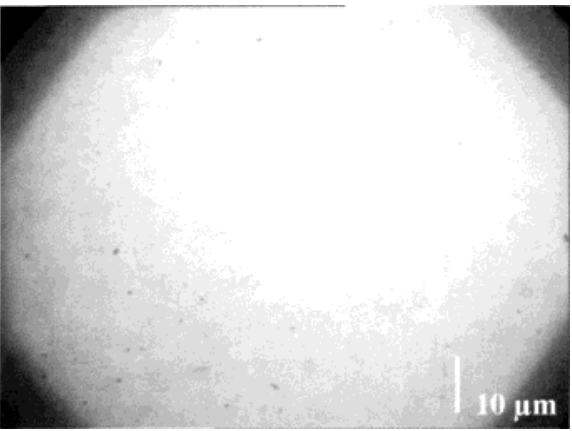

Figure 3. Fluorescence micrograph of a DMPC bilayer on a chitosan film, prepared by LB/LS transfer at a lateral pressure of $35 \mathrm{mN} / \mathrm{m}$ and deposition temperature of $15{ }^{\circ} \mathrm{C}$, using fluorescence probe NBD-PE.
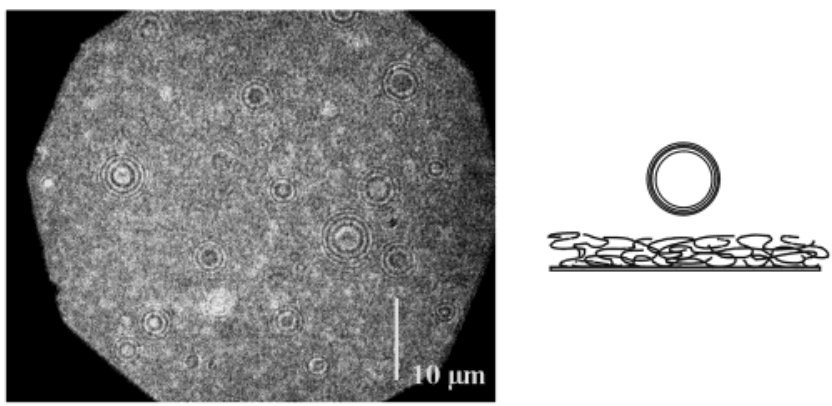

Figure 4. RICM image of DMPC vesicles hovering over an agarose surface (left); schematic drawing of a giant, multilamellar vesicle, hovering over the polymer surface (right).

observed. A successful bilayer preparation was possible only at the lower temperature. This was the case even though the lipid layer was still in the fluid state at the lower temperature, due to the additional cholesterol content (at a cholesterol content higher than $30 \mathrm{~mol} \%$, notransition toa solid phaseis found for DMPC/chol esterol mixtures ${ }^{47}$ ).

RICM Experiments. Agarose To further analyze the interaction between phospholipid bilayers and agarose, giant multilamel lar liposomes were added to the aqueous phase into which an agarose film was immersed. With the fluorescence mi croscopy results in mind, a strong adhesion of liposomes to the agarose surface could not be expected. Indeed, it was found by reflection interference contrast microscopy that li posomes werehovering over the agarose surface (Figure 4). Fast changes in height and position were observed, due to thermally activated movements of liposomes. ${ }^{48} \mathrm{Height}$ fluctuations wereindi cated by changes

(47) Almeida, P. F. F.; Vaz, W. L. C.; Thompson, T. E. Biochemistry 1992, 31, 6739-6741.

(48) Rädler, J .; Sackmann, E. Langmuir 1992, 8, 848-853.

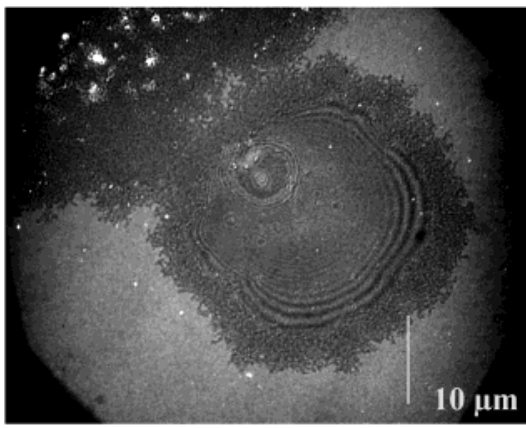

Figure 5. RICM image of giant multilamellar liposomes, partially spread on a chitosan surface at room temperature.

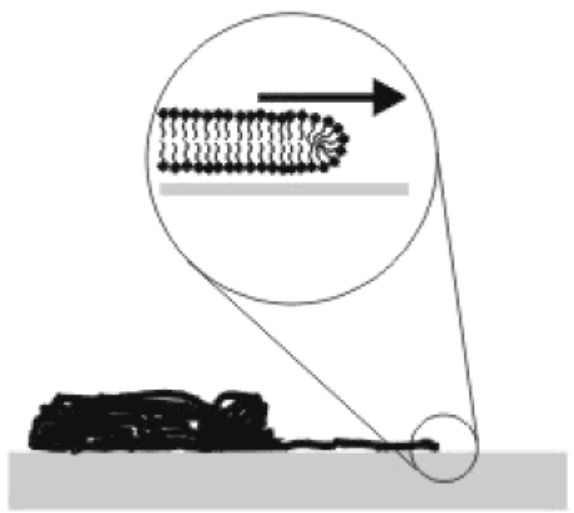

Figure 6. Bilayer spreading from a lipid reservoir, deposited onto a hydrophilic substrate from a solution. The substrate is immersed in water or a buffer solution afterward.

of the diameters of theinterferencefringes shown in Figure 4. Accordingly, the thermal energy was high enough to compensate for any attractive force between pol ymer and bilayer membrane, becausean adhesion of giant li posomes to an agarose surface was never observed.

Chitosan. According to the fluorescence microscopy observations described above, a higher interaction of chitosan with liposome surfaces is expected. Giant multilamellar liposomes consisting of DMPC were added to the aqueous phase above a chitosan film on a glass substrate. Indeed, an adsorption of liposomes was found. Liposomes, after the initial contact, flattened fast and finally ruptured. As multilamellar liposomes were used, the remaining lamellae could still be observed in the interferogram. Figure 5 shows an adsorption spot of a giant liposome on a chitosan surface. From Figure 5, it can be deduced that the polymer/liposome interaction is much stronger in thecase of chitosan compared to agar ose films. This stronger interaction explains the higher stability of planar chitosan-supported bilayer membranes. 

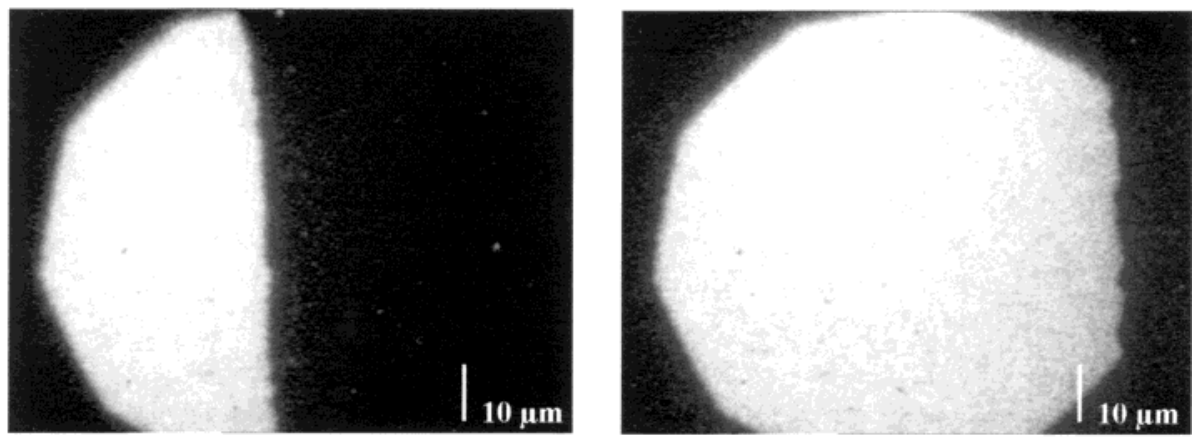

Figure 7. Fluorescence images of a spreading experiment on a chitosan surface. An egg-PC lipid reservoir was deposited onto the polymer film, and the substrate was immersed into MilliQ water. The right picture was taken 34 min after the left one.

Spreading Experiments. To further analyze the nature of theinteraction between bilayer lipid membranes and polysaccharidefilms, spreading fromalipid reservoir on polymer films was analyzed. The effect of singlebilayer spreading on planar surfaces was first observed by Rädler et al. ${ }^{1}$ In that work, a lipid reservoir was deposited from a solution onto inorganic surfaces (like glass or $\mathrm{MgF}_{2}$ ), thoroughly dried, and subsequently hydrated with water or buffer solution. Theresult was an advancement of single bilayers from thelipid reservoi $r$, as shown schematically in Figure6. L ater it was found that such bi layer spreading is also possible on extremely thin (in the range of $10 \mathrm{~nm}$ ), soft, and hydrated polymer cushions, such as dextran and cellulose. ${ }^{15}$ Both polymers wereassumed not tospecifically interact with lipid membranes. Therefore, theenergy gain by bilayer spreading was attributed to the van der Waals interaction between the membrane and the underlying (glass) substrate. Agaroseand chitosan polymer cushions were considerably thicker than the above-mentioned polymer layers. A much-reduced van der Waals contribution is therefore expected.

Whiletheadvancement of bilayers on agarosecushions was not observed (zwitterionic lipids were used in all cases), Figure 7 shows that thespreading of a singlebilayer froma lipid reservoir on chitosan is possible, which means that bilayers on chitosan possess self-heal ing properties. During theedge displacement of thebilayer, a roughening of the spreading front was observed (Figure 7).

To examine the kinetics of bilayer spreading, the time dependency of the edge displacement was analyzed. The length of the spreading monolayer was taken as the distance of the monolayer edge from the lipid reservoir (see Figure 6).

The kinetics of bilayer spreading can be described by means of the following equation: ${ }^{1}$

$$
\mathrm{v}(\mathrm{t})=\sqrt{\frac{\mathrm{W}_{\mathrm{A}}}{2 \zeta}} \frac{1}{\sqrt{\mathrm{t}}}=\sqrt{\frac{\beta}{\mathrm{t}}}
$$

where $\mathrm{W}_{\mathrm{A}}$ is the energy per unit area gained by spreading, $\xi$ is a viscous drag coefficient, and $\beta$ is a kinetic spreading coefficient. ${ }^{1}$ Hence plotting of $\log (v)$ against $\log (t)$ should yield a straight line with a slope of -0.5 .

From Figure 8, it can be deduced that a linear fit with a fixed slope of -0.5 successfully describes the spreading behavior on chitosan. From the interpolation to $\log (\mathrm{t})=$ 0 , the kinetic spreading coefficient was determined to be $\beta=1.8 \times 10^{-13} \mu \mathrm{m}^{2} / \mathrm{s}$.

Vesicle Fusion Experiments. Vesicle fusion onto hydrophobic or hydrophilic surfaces ${ }^{49,50}$ is a common

(49) Brian, A. A.; McConnell, H. M. Proc. Natl. Acad. Sci. U.S.A. 1984, 81, 6159-6163.

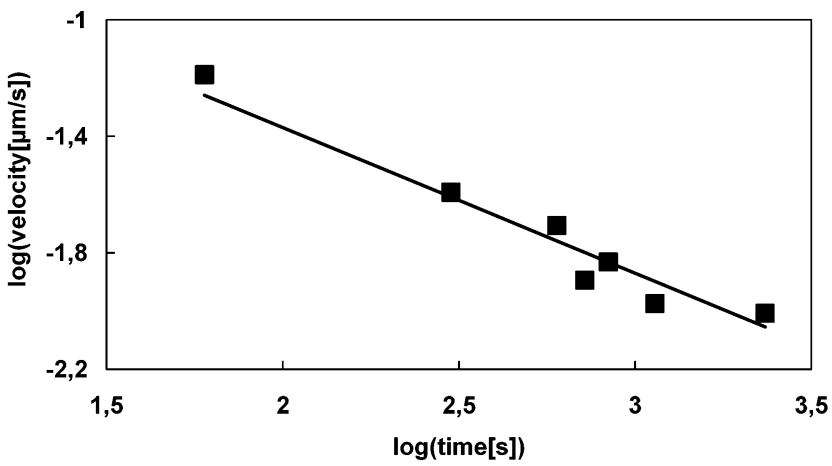

Figure 8. Double logarithmic pl ot of the spreading velocity against measurement time, for an egg-PC bilayer spreading on chitosan from a lipid reservoir; bold line, one-parameter fit with a fixed slope of -0.5 .

method for preparing sol id-supported bilayer membranes. The formation of laterally fluid bilayers by vesicle fusion on a cellulose surface was observed. ${ }^{51}$ However, using either agaroseor chitosan, vesicleadsorption did not result in laterally connected membranes. When agarose films weresubjected to a fluorescent-vesicledispersion, shaking thesubstratein pure water caused thefluorescence of the substrate to cease, implying that adhering vesicles were washed away. On chitosan films, adsorbed vesicles could not be washed away completely, but upon bleaching the fluorophores in a defined area (e.g., by means of intense laser light), nofluorescencerecovery, which would indicate lateral connectivity, was observed.

Lateral Diffusion on Chitosan. I n thecaseof agarose, no stable homogeneous membranes could be obtained by Langmuir-Blodgett trough methods. Chitosan-supported membranes, however, showed a sufficient stability for a characterization of membrane fluidity. By means of fluorescencemicroscopy, it was ensured that nomembrane fragmentation occurred during a measurement series.

Figure 9 shows the temperature dependence of lateral diffusion coefficients in lipid bilayers on chitosan and on glass in an Arrheni us plot, as obtained by photobleaching measurements (FRAP). From the comparison, the following conclusions may be drawn. The magnitude of the lateral fluidity was not significantly increased by the presence of a hydrated polymer cushion, compared to a glass support. Furthermore, whilethediffusion coefficients on chitosan- and glass-supported membranes were generally similar in magnitude, differences were found in the temperature region of the main phase transition of an

(50) Kalb, E.; Frey, S.; Tamm, L. K. Biochim. Biophys. Acta 1992 $1103,307-316$.

(51) Hillebrandt, H. Diploma Thesis, Technische Universität München, München, Germany, 2000. 


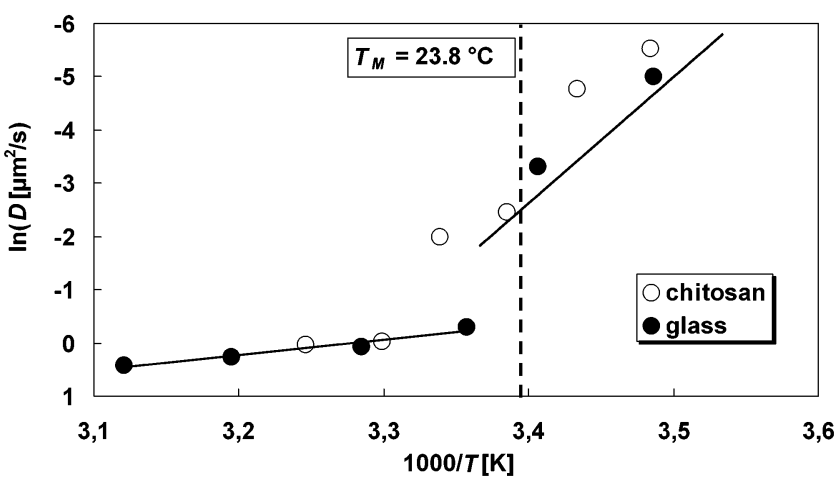

Figure 9. Comparison of the temperature-dependent lateral diffusion behavior of DMPC bilayer membranes labeled with NBD-PE, supported by gl ass and chitosan. In the case of glass, the phase transition as seen by lateral diffusion occurs around the phasetransition temperaturefound in nonperturbed DMPC bilayers (line). However, in the case of chitosan, the phase transition occurs at a higher temperature. Each data point represents the average of three to five single measurements.

unperturbed DMPC membrane. The main phase transition of unperturbed DMPC bilayers occurs at $23.8^{\circ} \mathrm{C} . .^{52}$ In the glass-supported bilayer, this first-order transition is indicated by a jump of the diffusion coefficients in an interval of $20.4-24{ }^{\circ} \mathrm{C}$ (Figure 9). This is in accordance with another report, ${ }^{53}$ where this phase transition was observed around $20^{\circ} \mathrm{C}$ (by FRAP experiments). A transition temperature close to the value for unperturbed bilayers was reported in ref 54 again for a glass-supported DMPC membrane.

However, in thecase of chitosan, thejump of D occurred in the interval of $26.4-30.0^{\circ} \mathrm{C}$, which indicates that the presence of chitosan causes an increase of the phase transition temperature.

\section{Discussion}

In the following discussion, we first examine the observation of the failure to produce planar supported bilayer membranes on agarose or chitosan films by the method of vesicl efusion of small unilamellar vesicles. We proceed with a detailed examination of the physicochemical parameters which influence membrane/substrate interactions.

VesicleF usion Experiments. A common phenomenon found with chitosan and agarose polymer cushions was the fact that small unilamellar vesicles did not fuse on the polymer surfaces to yield a laterally connected, supported bilayer membrane. In the case of agarose, adherent vesicles could easily be washed away by means of flushing with water which did not contain vesicles. This observation is in agreement with theconclusion that lipid membranes are not sufficiently attracted by thesubstrate in the case of agarose films. In the case of chitosan films, vesicles could not bewashed away completely, which again is in agreement with the conclusion that chitosan surfaces interact more strongly than agarose surfaces with lipid molecules bearing choline headgroups.

Wefound nofluorescencerecovery after photobleaching involving a chitosan film that was treated according to the vesicle fusion protocol described in the methods and materials section. This indicates adsorbed vesicles that did not open to form a laterally connected bilayer.

(52) Marsh, D. CRC Handbook of Lipid Bilayers; CRC Press: Boca Raton, FL, 1990.

(53) Merkel, R.; Sackmann, E.; Evans, E. J . Phys. France 1989, 50, 1535-1555.

(54) Tamm, L. K.; McConnell, H. M. Biophys. J . 1985, 47, 105-113.
The formation of planar supported lipid membranes by themethod of vesicl efusion has been described to proceed by the following sequence: vesicles approach the surface, adsorb to the surface initially, and either rupture or fuse with each other before rupture takes place.

Theformation of a homogeneous supported bilayer that covers the whole substrate surface was suggested to be due to a lateral spreading and fusion of a bilayer; ${ }^{1}$ on the other hand, it was proposed that a closed bilayer forms by the coalescence of single, substrate-supported bilayer patches which grow by thefusion of vesicles with thehighenergy edges of these bilayer patches. ${ }^{61}$ Since lateral spreading of li pid bilayer membranes fromalipid reservoir and theadsorption of vesicles on thechitosan surface were observed, weconcludethat theadsorbed small unilamellar vesicles did not rupture.

Physicochemical Parameters I nfluencing the Stability of Supported Bilayer Membranes. In the present work, a considerable difference of the stability of lipid bilayer membranes on agarose and on chitosan polysaccharide cushions was observed, while monolayer li pid membranes werefound to behomogeneous and stable in both cases.

To interpret these observations, it is necessary to separately examine the physicochemical parameters which influence membrane/substrate interactions.

Spreading Parameters of Mono- and Bilayer Membranes. Since a chemical fixation is absent, the stability of a substrate-supported, physisorbed membrane is determined by the spreading parameter, S, which is calculated from the interfacial energies, $\gamma$, of the surfaces indexed in eq 2.19

$$
\mathrm{S}=\gamma_{\text {polymer,water }}-\left(\gamma_{\text {polymer,lipid }}+\gamma_{\text {lipid,lipid }}+\gamma_{\text {lipid,water }}\right)
$$

The spreading power, S, has to be positive in the case of a stablemembrane. Since $\gamma_{\text {polymer, water }}$ for a highly hydrated polymer cushion is on the same order as $\gamma_{\text {water, water, which }}$ is zero, the spreading power is expected to be small or even negative. ${ }^{19}$ On the other hand, the stability of monolayers on a hydrated polymer support is much higher compared to that of bilayers, since in that case

$$
\mathrm{S}=\gamma_{\text {polymer,vapor }}-\left(\gamma_{\text {polymer,lipid }}+\gamma_{\text {lipid,vapor }}\right)
$$

and $\gamma_{\text {polymer,vapor }}$ is of thesame order as $\gamma_{\text {water,vapor. }}$. Ther efore thespreading power is positive. The deposition of a second monolayer onto a polymer-cushioned monolayer is a critical step, which, according to eq 2, may be followed by membrane decomposition.

By the complementary methods of fluorescence microscopy, reflection interference microscopy, and bilayer spreading, it was shown that membranes on chitosan were considerably morestablethan lipid membranes supported by agarose films.

Electrostatic Interactions. Theobserved higher stability of lipid bilayer membranes on chitosan might be due to the positive zeta potential of the chitosan film. The $\mathrm{pK}_{a}$ value of the glucosamine segments is in the range 6.37,55 which leads to a slight protonation in the case of the polymer immersed in MilliQ water $(\mathrm{pH}=5.5)$. Bilayers with cholineheadgroups, on theother hand, havea slightly negative electrostatic potential across the membrane/ water interface. This potential arises from an overcompensation of theli pid headgroup di pol epotential by a water dipolepotential, caused by theordering of water molecules

(55) Claesson, P. M.; Ninham, B. W. Langmuir 1992, 8, 1406-1412. 
near the bilayer surface. ${ }^{56} \mathrm{~F}$ urthermore, there might be an interaction of amino groups on the surface of chitosan films with the phosphategroup of the phosphatidylcholine headgroup. An additional electrostatic attraction arises by the use of the negatively charged membrane probe NBD-PE. However, it has been shown that chitosan forms a stable coating layer even on zwitterionic lipid vesicles. ${ }^{2}$ This behavior of chitosan is remarkably different from that of uncharged, water-solublepolymers. Poly(ethylene glycol) (PEG) for example does not bind to the surface of phosphatidylcholine membranes, and the exclusion from themembranesurfaceleads to a dehydration of liposomes, often accompanied by phase separations. ${ }^{57}$ While PEG acts as a liposome fusogen, chitosan-coated liposomes (chitosomes) are stable and the polymer coating does not desorb during washing by centrifugation. ${ }^{2}$

MembraneUndulations: TheE ffect of Cholesterol and theDeposition Temperature Even in the case of favorable electrostatic interactions, the membrane stability on a support may be considerably reduced by a disjoining pressure due to undulations, that is, thermally driven collective out-of-plane movements of the bilayer membrane, ${ }^{44,58}$

$$
P_{\text {und }}=\frac{3}{4} \frac{k T}{K_{c} d^{3}}
$$

where $\mathrm{P}_{\text {und }}$ is the disjoining pressure due to undulations, $\mathrm{k}$ is Boltzmann's constant, $\mathrm{T}$ is the temperature, $\mathrm{K}_{\mathrm{c}}$ is the bending modulus of the bilayer, and $\mathrm{d}$ is the membrane/ substrate distance. The bending modulus of a bilayer depends on the membrane phase state and is smaller in thecase of a membranein a fluid lamellar phase compared to a gel phase. ${ }^{64}$ This could lead to a higher stability of gel-phase membranes supported by a solid substrate, compared to a fluid membrane. As undulations are thermally excited, temperature reduction leads to a stabilization of thesupported membrane. This membrane stabilization is crucial, especially during the LangmuirSchäfer (LS) transfer, as strong flow in the subphase can cause bilayer rupture.

Another way of suppressing bilayer undulations is the addition of cholesterol, which was observed to enhance membranequality on a bilayer li pid membranesupported by dextran. ${ }^{19}$ However, in the case of agarose-supported bilayers containing cholesterol, vesiclebudding and fission were observed at the low deposition temperature of $15^{\circ} \mathrm{C}$ (Figure 2). This can be explained by the influence of cholesterol on the phase transition temperature of the bilayer. At $15^{\circ} \mathrm{C}$, a DMPC bilayer is in theliquid condensed state, while no transition to the liquid condensed state is found for a cholesterol content above $30 \mathrm{~mol} \% .{ }^{47}$ Another reason for an increased dewetting tendency upon addition of cholesterol is the comparably high roughness of the agarose cushion. ${ }^{59}$ While a bilayer with a low bending modulus could embed itself into a rough surfacestructure, this embedding becomes more difficult for a bilayer with an increased bending modulus (as is the case for cholesterol-containing bilayers). Theresult would bea decreased contact area and hence a decreased sticking of the membrane to the support.

(56) Marrink, S.-J .; Tieleman, D. P.; van Bueren, A. R.; Berendsen, H. J. C. Faraday Discuss. 1996, 103, 191-201.

(57) Lehtonen, J. Y. A.; Kinnunen, P. K. J . Biophys. J . 1995, 68, $525-535$.

(58) Helfrich, W. Z. Naturforsch. 1978, 33a, 305-315.

(59) Baumgart, T. Ph.D. Thesis, J ohannes Gutenberg Universität Mainz, Mainz, Germany, 2001.
Kinetic Spreading Coefficient. Bilayer spreading of a single membrane from a hydrated lipid reservoir allows characterizing membrane/support interactions. ${ }^{15}$ On agarose cushions, no advancement of a bilayer membrane was observed, while a spreading of a bilayer membrane was found on chitosan cushions (Figure 7). Thepossibility of bilayer spreading is considered to be important for practical applications of solid-supported bilayer lipid membranes, since it leads to self-healing properties of such systems. ${ }^{1}$ Thekinetic spreading coefficient of an eggPC bilayer membrane on chitosan at room temperature was determined to be $\beta=1.8 \times 10^{-13} \mu \mathrm{m}^{2} / \mathrm{s}$. This value is about 2 orders of magnitude smaller compared to the spreading of a DMPC bilayer on glass and 1 order of magnitudesmaller compared toDMPC bilayers spreading on cellulose or dextran films. ${ }^{15}$ The smaller value could beexplained by higher friction duetoa dehydration of the membrane/substrategap in thecase of chitosan-supported membranes (see below). Additionally, the driving force for spreading might be smaller compared to that for glass supports or ultrathin polymer films, since an energy gain by the van der Waals interaction between membraneand glass substrateis much smaller duetothemuch-increased bilayer/substrate separation distance (the chitosan films used in the present work had a thickness of $140 \mathrm{~nm}$ in the dry state). During the spreading of bilayers on chitosan surfaces, a roughening of the bilayer edge was observed by fluor escence microscopy. Such a roughening was also found in the case of spreading on glass surfaces and was attributed to line pinning, induced by the substrate roughness. ${ }^{1}$ This edge roughening is in contrast to monolayer spreading on the same substrates, where no significant line roughening was observed. ${ }^{60}$

The fact that no spreading of bilayers on agarose films was observed can, according to eq 1 , be explained by a small energy gain by bilayer spreading $W_{A}$, which is in accordance with the results from $L B$-trough experiments and RICM. However, theimpossibility of bilayer spreading on agarose cushions could also be due to a high drag coefficient $\xi$ on agarose films.

Temperature Influence on Membrane Fluidity. FRAP experiments revealed an increased main phasetransition temperature of a DMPC membranesupported by chitosan, as compared to data for free or glass-supported DMPC bilayer membranes (Figure 9). A similar effect can be observed in the case of DMPC bilayers supported by polyacrylamide films. ${ }^{19,62}$ However, differential scanning cal orimetry measurements of a mixture of multilamellar vesicles composed of DPPC and chitosan, immersed in a phosphate-buffered saline (PBS) buffer, provided no evidence for an increased main phase transition temperature due to DPPC/chitosan interactions. ${ }^{63}$ DPPC, however, has a higher main phase transition temperature than DMPC $\left(40^{\circ} \mathrm{C}^{63}\right.$ compared to $\left.23.8^{\circ} \mathrm{C}\right)$. The phospholipid/chitosan interaction is likely to be temperature dependent and might also depend on ionic strength. Therefore, the findings described above ${ }^{63}$ cannot be compared to our results.

An increase of the main phase transition temperature of the DMPC bilayer could be due to a slight dehydration of the membrane surface, relative to an unperturbed bilayer. This increase would then be a result of closer

(60) Baumgart, T.; Offenhäusser, A. Langmuir 2002, 18, 5899-5908.

(61) Reviakine, I.; Brisson, A. Langmuir 2000, 16, 1806-1815

(62) Pink, D.; Kühner, M.; Quinn, B.; Sackmann, E.; Pham, H. Langmuir 1995, 11, 2696-2704.

(63) Fang, N.; Chan, V.; Mao, H.-Q.; Leong, K. W. Biomacromolecules 2001, 2, 1161-1168.

(64) Duwe, H. P.; Sackmann, E. Physica A 1990, 163, 410-428. 
packing of lipid headgroups in theproximal leaflet, caused by headgroup dehydration. Such dehydration could bedue to the attractive chitosan/lipid interaction mentioned above.

\section{Conclusion}

Lipid bilayer membranes consisting of zwitterioniclipids and deposited onto agarose cushions by LangmuirBlodgett methods wereshown to be unstable. The bilayer instability was indicated by (a) membrane decomposition upon storage as observed by fluorescence microscopy, (b) missing adhesion of giant multilamel lar liposomes to the agarosesurfaceas measured by RICM, (c) theimpossibility of bilayer spreading from a lipid reservoi $r$ on the agarose surface, and (d) the fact that small unilamellar vesicles could becompletely washed away from theagar osesurface by gently shaking in pure water. In light of these observations, the stabilization of black lipid membranes by supporting them using an agarose cushion can only be regarded as a hydrodynamic one, in terms of the suppression of mechanical distortions.

Bilayer lipid membranes cushioned by thin chitosan films were shown to possess an increased stability compared to agarose-cushioned membranes. This higher stability was shown by (a) theobservation of homogeneous bilayer membranes by fluorescence microscopy, (b) a strong attraction of zwitterionic, giant unilamellar liposomes leading tovesiclerupture, and (c) thefact that single bilayer sliding on chitosan surfaces from a lipid reservoir deposited onto the polymer cushion was observed.

Chitosan-supported lipid membranes provide easy to prepare model membrane systems. They all ow for study of the bilayer itself and for the incorporation and examination of membrane proteins..$^{59} \mathrm{~F}$ undamental biological processes may be mimicked with the help of these supported model membrane systems. The advantages of the chitosan cushions described in the present work are simple preparation and controllablefilmthickness, which provides, for example, access to polymer-cushioned membranes for biological laboratories, wherefacilities al lowing for extensive chemical synthesis are missing. By the presence of a soft and hydrated polymer cushion, it is probable that integral membrane proteins may be incorporated into these model membrane systems in a functionally active form.

Acknowledgment. Wethank Wolfgang Knoll for his generous support within the project. We are indebted to AdamT. Hammond for a critical reading of our manuscript. T.B. was supported by the Graduiertenkolleg of the DFG.

LA0261489 